\title{
Clinical efficacy and problems with CT lymphography in identifying the sentinel node in breast cancer

\author{
Masako Takahashi ${ }^{1}$, Mitsunori Sasa*2, Chieko Hirose ${ }^{3}$, Sonoka Hisaoka ${ }^{3}$, \\ Masako Taki ${ }^{4}$, Toshiyuki Hirose ${ }^{5}$ and Yoshimi Bando ${ }^{6}$
}

\begin{abstract}
Address: ${ }^{1}$ Department of Radiology, Tokushima Breast Care Clinic, 4-7-7, Nakashimada-Cho, Tokushima, 770-0052, Japan, ${ }^{2}$ Department of Surgery, Tokushima Breast Care Clinic, 4-7-7, Nakashimada-Cho, Tokushima, 770-0052, Japan, ${ }^{3}$ Department of Radiology, National Higashi Tokushima Hospital, 1-1, Ohmukai-kita, Ootera, Itano, Tokushima, 779-0193, Japan, ${ }^{4}$ Department of Radiology, Tokushima Prefecture Hospital, 1-10-3, Kuramoto-cho, Tokushima, 770-8539, Japan, ${ }^{5}$ Department of Surgery, National Higashi Tokushima Hospital, 1-1, Ohmukai-kita, Ootera, Itano, Tokushima, 779-0193, Japan and ${ }^{6}$ Department of Molecular and Environmental Pathology, Institute of Health Biosciences, The University of Tokushima Graduate School, 3-18-15, Kuramoto-Cho, Tokushima, 770-8509, Japan

Email: Masako Takahashi - takapy55@tune.ocn.ne.jp; Mitsunori Sasa* - breast@mb.tcn.ne.jp;

Chieko Hirose - hirose@higashitokushima.hosp.go.jp; Sonoka Hisaoka - sonoka@mb3.tcn.ne.jp; Masako Taki - zaw00407@nifty.ne.jp;

Toshiyuki Hirose - toshi-hirose@higashitokushima.hosp.go.jp; Yoshimi Bando - yoshimi@basic.med.tokushima-u.ac.jp

* Corresponding author
\end{abstract}

Published: 12 June 2008

World Journal of Surgical Oncology 2008, 6:57 doi:10.1 186/1477-7819-6-57

This article is available from: http://www.wjso.com/content/6/1/57

(c) 2008 Takahashi et al; licensee BioMed Central Ltd.

This is an Open Access article distributed under the terms of the Creative Commons Attribution License (http://creativecommons.org/licenses/by/2.0), which permits unrestricted use, distribution, and reproduction in any medium, provided the original work is properly cited.

\begin{abstract}
Background: Combining a radioisotope with a dye-guided method is the best method for identification of the sentinel lymph nodes (SNs) in breast cancer. However, some institutions are limited to use of a dye-guided method alone. Recently, computed tomographic lymphography (CTLG) employing a nonionic contrast medium has achieved SN identification.
\end{abstract}

Patients and methods: 218 patients with primary breast cancer and no clinical evidence of lymph node metastasis were studied. SN identification was performed by CTLG and a dye-guided method. The SN identification rate was analyzed for correlations with the clinicopathological findings.

Results: The SN identification rates were $96 \%$ with CTLG, $92 \%$ with the dye-guided method and $99 \%$ with both methods combined. The identification rates with CTLG and the combined method were significantly lower in node-positive patients compared to node-negative patients, and significantly lower with the combined method in vascular invasion-positive patients compared to negative patients. In addition, the $\mathrm{SN}$ identification rate with the dye-guided method was significantly lower in patients with a body mass index (BMI) of $\geq 25$, whereas the BMI did not affect the identification rate with CTLG or the combined method. Multiple SNs were detected in approximately $20 \%$ of the patients.

Conclusion: Combined performance of CTLG and a dye-guided method enables identification of SNs prior to breast cancer surgery. That SN identification is easier compared with by the dyeguided method alone, and the identification rate is improved compared with either method alone. The combination of methods was especially useful in obese patients. For patients with multiple SNs, the combination has the further advantage of enabling accurate SN biopsy. CTLG may yield falsenegative findings in node-positive patients and patients with lymph vessel obstruction. 


\section{Background}

Sentinel node biopsy (SNB) has become a standard surgical procedure for patients with early-stage breast cancer $[1,2]$. The sentinel nodes (SN) can be identified by a double-mapping procedure based on a gamma probe-guided method and a dye-guided method using a radioisotope (RI), or by a triple-mapping procedure that includes lymphoscintigraphy and is even more effective [1-3]. However, the RI method can be performed only at institutions that are trained and licensed to use RI, and other institutions must rely on dye methods alone for $\mathrm{SN}$ identification [4-8] On the other hand, for obtaining images of the lymph vessels and nodes, indirect lymphography seems to be a more convenient than direct intralymphatic administration of a contrast medium. Several studies of indirect lymphography were reported in the 1980s [9-12], and Suga et al., reported successfully identifying $\mathrm{SN}$ by threedimensional computed tomographic lymphography (CTLG) using a nonionic contrast medium [6.7]. We have also been identifying SN in breast cancer patients by CTLG and a dye-guided method since February 2003 as a clinical trial [5]. Here, we report our findings to date regarding the clinical efficacy and problems associated with SN identification by the combination of CTLG and a dye-guided method.

\section{Patients and methods}

All studies in this paper were approved by the ethics committee of National Higashi-Tokushima Hospital. After presenting a detailed explanation of this clinical trial, written informed consent was obtained from all patients.

\section{Patients}

During the period from February 2003 through March 2007, 218 Japanese patients with T1NOMO or T2NOMO primary breast cancer were treated at the Tokushima Breast Care Clinic. SN identification was performed by combined application of CTLG and a dye-guided method. In two of the patients the CTLG was performed before and after excisional biopsy, and in one patient with bilateral disease CTLG was performed on both sides. Thus, CTLG was performed 221 times in total, while the dye-guided method was performed a total of 219 times. In principle, backup dissection was performed for patients found to be SN metastasis-positive provided that informed consent was granted. For metastasis-negative patients, axillary dissection was omitted on the basis of informed consent.

\section{Methods}

CTLG was performed as previously described [5]. Briefly, the CT apparatus was a high-speed FX/I single-detector helical CT scanner (GE Yokogawa Medical Systems, Tokyo, Japan). One $\mathrm{mL}$ of iopamidol (Iopamiron 300; $300 \mathrm{mgI} / \mathrm{ml}$, Schering, Osaka, Japan) was injected subcutaneously to the areola or both subcutaneously to the are- ola and subcutaneously directly above the tumor, and the $\mathrm{SN}$ was attempted to be identified by CT performed $1 \mathrm{~min}$ later. In the case that the enhancement of the lymph vessels and lymph nodes was poor, the site(s) where the contrast medium had been injected was massaged, and after 3-5 min the CT was repeated. SNs were predicted from CT images by identification of enhanced lymph vessels and/ or lymph nodes and assessment of the CT values. The CT values of lymph nodes were measured at their maximally enhanced point. If an SN(s) was identified, a mark was made on the skin immediately above the SN. The dyeguided method was also performed as previously described [5]. Briefly, 2 3 mL of indigo carmine blue was injected subcutaneously to the areola, followed by massage of the injection site for a few minutes. Just before performing an operation, we used ultrasonography to confirm the location of SN(s) that had been identified by CTLG. Five to $15 \mathrm{~min}$ later a skin incision was made at the site(s) that were marked in the CTLG, and any bluestained lymph vessels and lymph nodes were identified. If no SN(s) was identified by either the dye-guided method or CTLG, axillary lymph node dissection was performed.

\section{Definition of SNs}

CTLG: A lymph node was defined as an SN if visual inspection or the CT value (the increase of Hounsfield units after subcutaneous injection) confirmed it to be enhanced or if it was confirmed to connect to an enhanced lymph vessel.

Dye-guided method: A lymph node was defined as an SN if it was dyed blue or if it was confirmed to connect to a blue lymph vessel.

\section{SN identification rates and the clinicopathological findings} The results were analyzed in relation to the success/failure of $\mathrm{SN}$ identification and various clinicopathological parameters, including the patient age, menopausal status, body mass index (BMI), tumor diameter, presence/ absence of excisional biopsy, histological type, presence/ absence of lymph node metastasis and presence/absence of vascular invasion.

\section{Statistical analysis}

The relationships between the success/failure of SN identification and the clinicopathological findings were analyzed for statistical significance using the chi-square test. A $\mathrm{p}$ value of $<0.05$ was considered to indicate statistical significance.

\section{Results \\ SN identification rates by CTLG and the dye-guided method}

Identification of the SN(s) was achieved in 212 (96\%) of the total 221 performances of CTLG. The number of 
detected SNs ranged from 1 to 3, with a mean of 1.2 SNs. With the dye-guided method the identification rate was $92 \%$ (202/219 tests). The combined identification rate was 99\% (Table 1). With CTLG, both the lymph vessels and lymph nodes were clearly enhanced and the SNs could be identified in 189 patients (86\%). In 5 patients (2\%) the lymph vessels were not enhanced but the lymph nodes were, and the SNs could be identified. In 7 other patients (3\%), the lymph vessels were enhanced while the lymph nodes were not clearly enhanced, but the SNs could be identified from the CT value. In 5 patients $(2 \%)$ neither the lymph vessels nor the lymph nodes were enhanced, but the SNs could be identified from the CT value. In 6 patients (3\%) the lymph vessels were enhanced but the lymph nodes were not, but the SNs could be identified on the basis of confirmation of their connection to the enhanced lymph vessels. Finally, in 9 patients (4\%) neither the lymph vessels nor the lymph nodes were enhanced, and SNs could not be demonstrated (Table 2).

SN identification rates and the clinicopathological findings Analysis of the relationships between the SN identification rates and the clinicopathological findings showed that the SN identification rates with CTLG, the dye-guided method and the combined method showed no differences as a function of the age, menopausal status, tumor diameter or histopathological type. However, with the dyeguided method the SN identification rate was significantly lower in patients with a BMI of 25 or higher, whereas with CTLG and the combined method the SN identification rate was not influenced by the BMI. With CTLG and the combined method, the SN identification rate was significantly lower in the node-positive patients, and with the combined method it was significantly lower in patients with vascular invasion. In addition, in the patients who had undergone lateral-upper region excisional biopsy the SN identification rate with CTLG was lower than in the patients who had undergone excisional biopsy in a region other than the lateral-upper region, although the difference did not reach statistical significance. Moreover, with the dye-guided method the SN identification rate was lower in the patients who had undergone excisional biopsy, regardless of the region, compared with the patients who had not undergone excisional biopsy (Table 3).

Table I: Identification rates of lymph vessels and sentinel lymph nodes

\begin{tabular}{llll}
\hline & Dye & CTLG & Combination \\
\hline Lymph vessels & $212(97 \%)$ & $202(91 \%)$ & $215(98 \%)$ \\
Sentinel lymph node & $202(92 \%)$ & $212(96 \%)$ & $216(99 \%)$ \\
\hline
\end{tabular}

Dye : Dye-guided method

CTLG : CT Lymphography

Combination: Combination of CTLG and Dye
Table 2: Findings of CT lymphography $n=221$

\begin{tabular}{lll}
\hline Lymph vessels & Nodes & \\
\hline Enhanced & Clearly enhanced & $189(86 \%)$ \\
Unenhanced & Clearly enhanced & $5(2 \%)$ \\
Enhanced & Assessment of CT values & $7(3 \%)$ \\
Unenhanced & Assessment of CT values & $5(2 \%)$ \\
Enhanced & Unenhanced & $6(3 \%)$ \\
unenhanced & unenhanced & $9(4 \%)$ \\
\hline
\end{tabular}

\section{Size of the metastasis and the $\mathrm{SN}$ identification rate in node-positive patients}

The patients found to be positive for metastasis were classified and analyzed on the basis of the size of the metastasis: $<0.2 \mathrm{~mm}, 0.2 \sim 2 \mathrm{~mm}$ and $>2 \mathrm{~mm}$. The results showed that the SN identification rate by both CTLG and the dyeguided method decreased as the size of the metastasis increased, although the difference did not reach statistical significance (Table 4).

\section{Number of lymph vessels leading to SNs and the number of SNs}

In this study, we were able to analyze the data on the lymph vessels leading to SNs and the number of SNs of 200 patients. A single route with a single SN was the most common pattern, seen in $68 \%$ of the patients. Multiple routes with a single SN were detected in $4 \%$ of the patients, while a single route with multiple SNs was seen in $10 \%$ and multiple routes with multiple SNs were seen in $8 \%$. Overall, multiple SNs were identified in $18 \%$ of the patients (Table 5).

\section{Discussion}

In breast cancer surgery, dissection of the axillary lymph nodes is considered effective for the objectives of performing staging and achieving local control $[1,13]$. Therefore, in recent years SNB has become a standard procedure for patients with no metastasis of the axillary lymph nodes since it avoids unnecessary dissection [1,2]. Combined use of an RI (gamma probe-guided method and lymphoscintigraphy) and a dye is currently considered to be more efficient for identification of the $\mathrm{SN}(\mathrm{s})$ than single use of either of these methods [1-3]. In Japan, there are many institutions that do not have the necessary facilities for using RIs and thus must use the dye-guided method alone to identify SNs $[4,5]$. On the other hand, Suga et al. proposed using a method called CTLG, which employs a nonionic contrast medium $[6,7]$, and we have applied that method for SN identification since February 2003 [5]. In this paper we have reported our findings regarding the usefulness and problems associated with combined application of CTLG and the dye-guided method for SN identification. 
Table 3: Sentinel node (s) identification rate and clinicopathological findings (all cases) (Dye $n=219$, CTLG $n=221$, Combination $n=$ 219)

\begin{tabular}{|c|c|c|c|}
\hline & Dye $(+)(n=202)$ & CTLG $(+)(n=212)$ & Combination $(+)(n=216)$ \\
\hline \multicolumn{4}{|l|}{ Age, years } \\
\hline$\leqq 35$ & $6(100 \%)$ & $6(100 \%)$ & $6(100 \%)$ \\
\hline $36 \sim 50$ & $87(90 \%)$ & $92(95 \%)$ & $96(98 \%)$ \\
\hline$\geqq 5 \mathbf{I}$ & $109(94 \%)$ & $114(97 \%)$ & $114(98 \%)$ \\
\hline \multicolumn{4}{|l|}{ Menopausal state } \\
\hline Pre & $103(92 \%)$ & $108(96 \%)$ & $105(98 \%)$ \\
\hline Post & $99(93 \%)$ & 104(95\%) & III(99\%) \\
\hline \multicolumn{4}{|l|}{ BMI } \\
\hline$<25$ & $160(95 \%) \#$ & $164(96 \%)$ & $166(98 \%)$ \\
\hline$\geqq 25$ & $42(84 \%)$ & $48(96 \%)$ & $50(100 \%)$ \\
\hline \multicolumn{4}{|l|}{ Tumor size, cm } \\
\hline$\leqq \mathbf{2 . 0}$ & $183(92 \%)$ & 193(96\%) & 197(99\%) \\
\hline$\geqq 2.1$ & $19(100 \%)$ & $19(100 \%)$ & $19(100 \%)$ \\
\hline \multicolumn{4}{|l|}{ With excisionary biopsy* } \\
\hline In lateral-upper region & I7(89\%) & $18(95 \%)$ & $19(100 \%)$ \\
\hline In other regions & $46(88 \%)$ & $52(100 \%)$ & $52(100 \%)$ \\
\hline \multicolumn{4}{|l|}{ Histological type } \\
\hline Ilal & $7 \mathrm{l}(88 \%)$ & $80(99 \%)$ & $80(99 \%)$ \\
\hline Ila2 & $22(100 \%)$ & $23(100 \%)$ & $22(100 \%)$ \\
\hline Ila3 & $64(96 \%)$ & $63(94 \%)$ & $65(97 \%)$ \\
\hline Ilb3 & $8(89 \%)$ & $8(89 \%)$ & $9(100 \%)$ \\
\hline DCIS & $13(100 \%)$ & $12(92 \%)$ & $13(100 \%)$ \\
\hline Others & $24(89 \%)$ & $26(93 \%)$ & $27(100 \%)$ \\
\hline \multicolumn{4}{|l|}{ Nodal status } \\
\hline n(-) & $177(93 \%)$ & 189(98\%) \# & $191(100 \%) \#$ \\
\hline$n(+)$ & $25(89 \%)$ & $23(82 \%)$ & $25(89 \%)$ \\
\hline \multicolumn{4}{|l|}{ Vascular invasion } \\
\hline $\mathbf{v}(-)$ & $160(91 \%)$ & $172(97 \%)$ & 174(99\%) \# \\
\hline$v(+)$ & $42(95 \%)$ & $40(91 \%)$ & $42(95 \%)$ \\
\hline
\end{tabular}

BMI : Body mass index

Dye : Dye-guided method

CTLG : CT Lymphography

Combination: Combination of CTLG and Dye

\#; $\mathrm{P}<0.05$

*; cases with excisional biopsy $(\mathrm{n}=7 \mathrm{I})$

Our experimental subjects were 219 Japanese patients with primary breast cancer that was thought to be clinically free of axillary lymph node metastasis. CTLG and the dye-guided method were employed in an attempt to identify the SN(s) in each of the patients. The SN identification rates were 96\% with CTLG alone, 92\% with the dyeguided method alone and $99 \%$ when the findings with the two methods were combined. Those results are good when compared with the published data for combined use of an RI and the dye-guided method [2,3]. In particu-

Table 4: Size of the metastatic lesion in the node positive cases $n$ $=\mathbf{2 8}$

\begin{tabular}{lll}
\hline Metastatic lesion size & Dye(+) & CTLG(+) \\
\hline Isolated tumor cells(<0.2 mm) & $2(100 \%)$ & $2(100 \%)$ \\
Micrometastasis(0.2 2 $\mathbf{~ m m})$ & $10(91 \%)$ & $9(82 \%)$ \\
Macrometastasis( $\geqq 2 ~ \mathbf{~ m})$ & $13(87 \%)$ & $12(80 \%)$ \\
\hline
\end{tabular}

Dye: Dye-guided method, CTLG: CT Lymphography lar, our SN identification rate with the dye-guided method is better than the rates that have been reported to date $[2,3,14]$. We think that this is because the location of the SN had already been determined by the CTLG, making it easy to identify the lymph vessels and lymph nodes. The breakdown of the identification by CTLG showed clear enhancing of the lymph nodes in $88 \%$ of the patients, while in $5 \%$ of the patients identification of the SN was possible on the basis of the CT value in spite of the fact that the lymph nodes were not clearly enhanced. Because the observations were macroscopic, when the dye-guided

Table 5: Number of lymph vessels leading to Sentinel nodes and the number of Sentinel nodes

\begin{tabular}{ll}
\hline Single rout and Single node & $151(68 \%)$ \\
Multi routs and single node & $8(4 \%)$ \\
Single rout and multi nodes & $23(10 \%)$ \\
Multi routs and multi nodes & $18(8 \%)$ \\
\hline
\end{tabular}


method was used alone, SN identification was difficult unless the lymph node was stained to a sufficient degree. With CTLG, on the other hand, even if the enhancement is not very striking it can be surmised that the SN identification rate will be improved since the CT value can be taken into consideration.

In 14 patients, the SN was identified by CTLG, but not by the dye-guided method. The location of the SN was confirmed by ultrasonography just before the operation in all of these patients. In these 14 patients, not only the SN but also neighboring nodes were sampled at the same time. Therefore, it seemed that the SN biopsies had been performed accurately.

The sensitivity of CTLG could not be investigated in our patient population because backup axillary dissection was not performed for patients who were metastasis-negative in the SNB. In addition, none of the patients experienced postoperative recurrence in the axillary lymph nodes, but that does not serve as a basis for claiming that there were few false-negatives [1]. However, Tangoku et al., reported the sensitivity of CTLG to be $98 \%$, and for that reason it can be thought that the sensitivity of CTLG is not inferior to that of the RI method [15].

With regard to correlations between the clinicopathological findings and the $\mathrm{SN}$ identification rate, the patient age, the location of the tumor, whether or not surgical biopsy was performed, the tumor size and the presence/absence of lymph node metastasis have been reported to influence identification of the SN [1,16-18]. In our patient series, the results showed no differences as a function of the age, menopausal status, tumor diameter or histopathological type. However, with the dye-guided method the SN identification rate was significantly lower in patients with a BMI of 25 or higher, whereas with CTLG and the combined method the SN identification rate was not influenced by the BMI. As the reason for this difference it is noted that with the dye-guided method it can be difficult to discern staining of lymph vessels and nodes with the naked eye if considerable subcutaneous fat is present, whereas with CTLG the subcutaneous fat plays no role since the observation is done by CT. In addition to the BMI, discrepancies between the SN identification rates with these three methods were observed as a function of the presence/absence of lymph node metastasis, the site of excisional biopsy (i.e., the lateral upper region and regions other than the lateral upper region), and the presence/absence of vascular invasion. Especially, the identification rates with CTLG and the combined method were significantly lower in node-positive patients compared to node-negative patients, and significantly lower with the combined method in vascular invasion-positive patients compared to negative patients. The size of injected parti- cles, the injected volume and the injection site have been reported as examination factors that can influence SN identification [2]. The nonionic contrast media that are used in CTLG have a larger particle size than dyes [19]. For that reason it can be hypothesized that, in patients with lymph node metastasis or vascular invasion and in patients who had undergone lateral upper region excisional biopsy, who can be predicted to have lymph vessel occlusion, the movement of a nonionic contrast medium would be impeded compared with that of a dye, thus resulting in a lower $\mathrm{SN}$ identification rate. On the other hand, the SN identification rate with the dye-guided method was lower in the patients with excisional biopsy in any region than in patients without excisional biopsy. It can be hypothesized that this is probably because excisional biopsy leads to edema of the connective tissue, which makes it difficult to distinguish the dye.

We also investigated the number of SNs and the number of lymph vessels leading to them [20-22]. These evaluations are difficult to achieve by the gamma probe-guided method and the dye-guided method, but CTLG permits detailed investigation. Our results showed that there was a single route leading to a single $\mathrm{SN}$ in $68 \%$ of the patients, multiple routes leading to a single $\mathrm{SN}$ in $4 \%$, a single route leading to multiple SNs in $10 \%$ and multiple routes leading to multiple SNs in $8 \%$. These results are in agreement with those reported by Tangoku et al., [23]. Thus, approximately $18 \%$ of the patients in our series had multiple SNs, and it can be hypothesized that it would be difficult to biopsy all of them if only the dye-guided method were employed. We think that combined use of CTLG with the dye-guided method would permit accurate biopsy, and for that reason we anticipate that combined performance of CTLG will prove useful.

CTLG is a diagnostic test that is performed prior to surgery for breast cancer. Studies are warranted to determine whether the CT findings or performance of fine-needle aspiration cytology of the SN will make it possible to achieve diagnosis of $\mathrm{SN}$ metastasis preoperatively and then decide whether or not SNB should be performed [24]. Such diagnosis leading to avoidance of unnecessary surgical procedures would represent a great clinical advantage by reducing the burden on the patient. Moreover, in the future it will be necessary to compare the usefulness of CTLG with the RI method.

\section{Conclusion}

Combined performance of CTLG with the dye-guided method permits better elucidation of the location of the $\mathrm{SN}(\mathrm{s})$ in breast cancer. This makes it easier to identify the $\mathrm{SN}(\mathrm{s})$ and results in a higher SN identification rate compared with application of the dye-guided method alone. Combination of CTLG and the dye-guided method was 
especially useful in obese patients. In addition, in patients with multiple SNs, it is advantageous to be able to perform SNB accurately. However, in patients with occlusion of the lymph vessels due to lymph node metastasis, having undergone lateral upper region lumpectomy or the presence of vascular invasion, there is a possibility that false-negative diagnostic results will be generated with CTLG.

\section{Abbreviations}

SN: sentinel node; SNB: sentinel node biopsy; CTLG: computed tomographic lymphography; BMI: body mass index; RI: radioisotope.

\section{Competing interests}

The authors declare that they have no competing interests.

\section{Authors' contributions}

MS initiated and co-wrote the paper with MT and $\mathrm{CH}, \mathrm{YB}$ examined surgical specimen, MS, SH, MT and TH took part in the care of patients and helped in preparation of the manuscript. All authors read and approved the manuscript.

\section{References}

I. Veronesi U, Paganelli G, Viale G, Luini A, Zurrida S, Galimberti V, Intra M, Veronesi P, Maisonneuve P, Gatti G, Mazzarol G, De Cicco C, Manfredi G, Fernández JR: Sentinel-lymph-node biopsy as a staging procedure in breast cancer: update of a randomised controlled study. Lancet Oncol 2006, 7:983-990.

2. Motomura K, Egawa C, Komoike Y, Kataoka T, Nagumo S, Koyama $\mathrm{H}$, Inaji $\mathrm{H}$ : Sentinel node biopsy for breast cancer: technical aspects and controversies. Breast Cancer 2007, I 4:25-30.

3. Giuliano $A E$, Jones $R C$, Brennan M, Statman R: Sentinel lymphadenectomy in breast cancer. J Clin Oncol 1997, 15:2345-2350.

4. Minato M, Hirose C, Sasa M, Nishitani H, Hori A, Morimoto T: Axillary 3D CT imaging with lymphoscintigraphy is useful for sentinel node biopsy in breast cancer. Anticancer Res 2003 , 23:2935-2940

5. Minato M, Hirose C, Sasa M, Nishitani H, Hirose Y, Morimoto T: 3dimensional computed tomography lymphography-guided identification of sentinel lymph nodes in breast cancer patients using subcutaneous injection of nonionic contrast medium: a clinical trial. J Comput Assist Tomogr 2004, 28:46-5 I.

6. Suga K, Ogasawara N, Okada M, Matsunaga N: Interstitial CT lymphography-guided localization of breast sentinel lymph node: preliminary results. Surgery 2003, I33:170-I79.

7. Suga K, Ogasawara N, Yuan Y, Okada M, Matsunaga N, Tangoku A: Visualization of breast lymphatic pathways with an indirect computed tomography lymphography using a nonionic monometric contrast medium iopamidol: preliminary results. Invest Radiol 2003, 38:73-84.

8. Cox CE, Pendas S, Cox JM, Joseph E, Shons AR, Yeatman T, Ku NN, Lyman GH, Berman C, Haddad F, Reintgen DS: Guidelines for sentinel node biopsy and lymphatic mapping of patients with breast cancer. Ann Surg 1998, 227:645-653.

9. Musumeci R, Tesoro-Tess JD, Costa A, Veronesi U: Indirect lymphography of the breast with iotasul: a vanishing hope? Lymphology 1984, 17:118-123.

10. Weissleder H, Weissleder R: Interstitial lymphangiography: initial clinical experience with a dimeric nonionic contrast agent. Radiology 1989, 170:37|-374.

II. Partsch H, Urbanek A, Wenzel-Hora B: The dermal lymphatics in lymphoedema visualized by indirect lymphography. $\mathrm{Br} J \mathrm{Der}-$ matol 1984, I I 0:431-438.
12. Wenzel-Hora BI, Kalbas B, Siefert HM, Arndt JO, Schlösser HW, Huth F: lotasul, a water-soluble (non-oily) contrast medium for direct and indirect lymphography: radiological and morphological investigations in dogs. Lymphology I98I, I4:I0I-II2.

13. Fisher B, Jeong JH, Anderson S, Bryant J, Fisher ER, Wolmark N: Twenty-five-year follow-up of a randomized trial comparing radical mastectomy, total mastectomy, and total mastectomy followed by irradiation. N Engl J Med 2002, 347:567-575.

14. Giuliano AE: Sentinel lymphadenectomy in primary breast carcinoma: an alternative to routine axillary dissection. I Surg Oncol 1996, 62:75-77.

15. Giuliano AE: Lymphatic mapping and sentinel node biopsy in breast cancer. JAMA 1997, 277:791-792.

16. Tangoku A, Yamamoto S, Suga K, Ueda K, Nagashima Y, Hida M, Sato T, Sakamoto K, Oka M: Sentinel lymph node biopsy using computed tomography-lymphography in patients with breast cancer. Surgery 2004, 135:258-265.

17. Noguchi M, Motomura K, Imoto S, Miyauchi M, Sato K, Iwata H, Ohta $M$, Kurosumi $M$, Tsugawa $K$ : A multicenter validation study of sentinel lymph node biopsy by the Japanese Breast Cancer Society. Breast Cancer Res Treat 2000, 63:3I-40.

18. Feldman SM, Krag DN, McNally RK, Moor BB, Weaver DL, Klein P: Limitation in gamma probe localization of the sentinel node in breast cancer patients with large excisional biopsy. J Am Coll Surg 1999, I 88:248-254.

19. Ollila DW, Giuliano AE: Intraoperative lymphatic mapping and sentinel lymphadenectomy using isosulphan blue dye. Breast Dis 1998, 8:297-300.

20. De Cicco C, Chinol M, Paganelli G: Intraoperative localization of the sentinel node in breast cancer: technical aspects of lymphoscintigraphic methods. Semin Surg Oncol 1998, 15:268-27I.

21. Duncan M, Cech A, Wechter D, Moonka R: Criteria for establishing the adequacy of a sentinel lymphadenectomy. Am J Surg 2004, 187:639-642.

22. Doting MH, Jansen L, Nieweg OE, Piers DA, Tiebosch AT, Koops HS, Rutgers EJ, Kroon BB, Peterse JL, Olmos RA, de Vries J: Lymphatic mapping with intralesional tracer administration in breast carcinoma patients. Cancer 2000, 88:2546-2552.

23. Leong SPL: Selective sentinel lymph node mapping and dissection for malignant melanoma.: "Atlas of selective sentinel lymphadenectomy for melanoma, breast cancer and colon cancer.". Edited by: Leong SPL. Boston: Kluwer Academic; 2002:39-64.

24. Tangoku A, Seike J, Nakano K, Nagao T, Honda J, Yoshida T, Yamai $\mathrm{H}$, Matsuoka H, Uyama K, Goto M, Miyoshi T, Morimoto T: Current status of sentinel lymph node navigation surgery in breast and gastrointestinal tract. J Med Invest 2007, 54:I-I8.

25. Nori J, Vanzi E, Bazzocchi M, Bufalini FN, Distante V, Branconi F, Susini T: Role of axillary ultrasound examination in the selection of breast cancer patients for sentinel node biopsy. Am J Surg 2007, 193:16-20.
Publish with Bio Med Central and every scientist can read your work free of charge

"BioMed Central will be the most significant development for disseminating the results of biomedical research in our lifetime. "

Sir Paul Nurse, Cancer Research UK

Your research papers will be:

- available free of charge to the entire biomedical community

- peer reviewed and published immediately upon acceptance

- cited in PubMed and archived on PubMed Central

- yours - you keep the copyright 